Article

\title{
Inter-Individual Differences Explain More Variance in Conditioned Pain Modulation Than Age, Sex and Conditioning Stimulus Intensity Combined
}

\author{
Philipp Graeff ${ }^{1,2, *}$, Alina Itter ${ }^{3}$, Katharina Wach ${ }^{3}$ and Ruth Ruscheweyh 1,2,3 \\ 1 Graduate School of Systemic Neuroscience, Ludwig-Maximilians-University Munich, \\ 82152 Planegg, Germany; ruth.ruscheweyh@med.uni-muenchen.de \\ 2 Research Training Group (RTG) 2175 Perception in Context and Its Neural Basis, \\ Ludwig-Maximilians-University Munich, 82152 Planegg, Germany \\ 3 Department of Neurology, University Hospital Großhadern, Ludwig-Maximilians-University Munich, \\ 81377 Munich, Germany; a.itter@campus.lmu.de (A.I.); katharina.wach@gmx.de (K.W.) \\ * Correspondence: Philipp.Graeff@campus.lmu.de
}

check for updates

Citation: Graeff, P.; Itter, A.; Wach, K.; Ruscheweyh, R. Inter-Individual Differences Explain More Variance in Conditioned Pain Modulation Than Age, Sex and Conditioning Stimulus Intensity Combined. Brain Sci. 2021, 11, 1186. https://doi.org/10.3390/ brainsci11091186

Academic Editor:

Elena Enax-Krumova

Received: 31 July 2021

Accepted: 6 September 2021

Published: 9 September 2021

Publisher's Note: MDPI stays neutral with regard to jurisdictional claims in published maps and institutional affiliations.

Copyright: (c) 2021 by the authors. Licensee MDPI, Basel, Switzerland. This article is an open access article distributed under the terms and conditions of the Creative Commons Attribution (CC BY) license (https:/ / creativecommons.org/licenses/by/ $4.0 /)$.

\begin{abstract}
Conditioned pain modulation (CPM) describes the reduction in pain evoked by a test stimulus (TS) when presented together with a heterotopic painful conditioning stimulus (CS). CPM has been proposed to reflect inter-individual differences in endogenous pain modulation, which may predict susceptibility for acute and chronic pain. Here, we aimed to estimate the relative variance in CPM explained by inter-individual differences compared to age, sex, and CS physical and pain intensity. We constructed linear and mixed effect models on pooled data from 171 participants of several studies, of which 97 had repeated measures. Cross-sectional analyses showed no significant effect of age, sex or CS intensity. Repeated measures analyses revealed a significant effect of CS physical intensity $(p=0.002)$ but not CS pain intensity $(p=0.159)$. Variance decomposition showed that inter-individual differences accounted for $24 \%$ to $34 \%$ of the variance in CPM while age, sex, and CS intensity together explained $<3 \%$ to $12 \%$. In conclusion, the variance in CPM explained by inter-individual differences largely exceeds that of commonly considered factors such as age, sex and CS intensity. This may explain why predictive capability of these factors has had conflicting results and suggests that future models investigating them should account for inter-individual differences.
\end{abstract}

Keywords: conditioned pain modulation; endogenous analgesia; conditioning stimulus; interindividual factors; $\mathrm{CPM}$ variability

\section{Introduction}

Conditioned pain modulation (CPM) paradigms measure the component of human endogenous pain inhibition underlying the "pain inhibits pain" phenomenon [1], based on a noxious test stimulus (TS) being perceived as less painful if presented in combination with a painful heterotopic conditioning stimulus (CS). CPM magnitude is reduced in a variety of chronic pain conditions, pointing towards dysregulation of endogenous pain inhibition in these patients [2].

Individual differences in CPM are considerable, and have been proposed to predict susceptibility to acute and chronic pain [3,4]. Some individual factors influencing CPM magnitude have been identified: e.g., some studies have found a larger CPM effect in males than females [5,6] and in younger compared to older subjects [7,8]. An effect of pre-existing psychological factors has been discussed, but a recent study has not shown a clear relation to the CPM effect [9]. It is currently not known how much individual variance remains after accounting for the effects of age and sex.

In addition, many different experimental paradigms have been used [10] which may also influence CPM magnitude., e.g., the role of conditioning stimulus intensity has been 
investigated repeatedly with inconsistent results. While some studies find no effect [11-13], others find larger CPM with stronger conditioning stimuli [14-16]. It has been proposed that as long as it is clearly painful, further increases in conditioning stimulus intensity do not increase CPM magnitude [12,13]. It might also occur that conditioning stimulus intensity does have an effect in within-subject designs [14,17], but that the effect is small compared to inter-individual differences, which makes it difficult to detect in cross-sectional designs. In addition, it is worth considering whether physical stimulus intensity or rather subjective pain perception of the conditioning stimulus is related to CPM magnitude.

It would therefore be useful to estimate the relative importance of the various factors influencing the CPM effect. There are now methods to estimate variance contributions of both fixed effects (such as sex, age and conditioning stimulus intensity) and random effects (such as remaining individual differences) within the same model [18,19], in addition to the relative variance contributions of the different fixed effects [20,21].

Here, we used pooled datasets from various studies measuring the CPM effect in healthy individuals once or multiple times to assess the relationship between CPM effect and age, sex, and conditioning stimulus physical or pain intensity in both cross-sectional and repeated measures settings, and estimated the relative variance in CPM magnitude explained by remaining inter-individual differences vs. age, sex, and conditioning stimulus intensity.

\section{Materials and Methods}

\subsection{Pooled Data}

Data was pooled from seven separate studies performed by our group, which investigated different aspects of endogenous pain inhibition in healthy participants, including at least one measurement of the conditioning pain modulation effect. Four studies included repeated measures gathered on different days. In total, data was pooled from 171 participants for cross-sectional analysis and from 97 participants for repeated-measures analysis. Of the repeated measures data pool, 83 participants had two repeated measures and 14 participants had three repeated measures.

Pooled data included three types of test stimulus: a $30 \mathrm{~s}$ or $60 \mathrm{~s}$ heat stimulus or electrical stimulation of the sural nerve. Conditioning stimulus in all studies was a cold pressor test of varying length $(60 \mathrm{~s}, 90 \mathrm{~s}, 120 \mathrm{~s})$. An overview of the studies can be found in Table 1.

Table 1. Overview of studies used for pooled data in this analysis. $\mathrm{N}=171$ participants total. Repeated measures: $\mathrm{n}=97$ participants, $\mathrm{n}=208$ observations.

\begin{tabular}{|c|c|c|c|c|c|c|}
\hline Study & Age & $\mathbf{M} / \mathbf{F}$ & $\begin{array}{l}\text { Conditioning } \\
\text { Stimulus }\end{array}$ & $\begin{array}{c}\text { Test } \\
\text { Stimulus }\end{array}$ & $\begin{array}{l}\text { Repeated } \\
\text { Measures }\end{array}$ & Citation \\
\hline 1 & $25 \pm 6$ & $18 / 12$ & Cold water (120 s) & Electrical & Yes & Unpublished \\
\hline 2 & $23 \pm 4$ & $15 / 5$ & Cold water (60 s) & $\begin{array}{l}\text { Contact } \\
\text { heat }(30 \mathrm{~s})\end{array}$ & Yes & Unpublished \\
\hline 3 & $27 \pm 6$ & $14 / 9$ & Cold water (60 s) & $\begin{array}{c}\text { Contact } \\
\text { heat }(30 \mathrm{~s})\end{array}$ & Yes & [22] \\
\hline 4 & $47 \pm 10$ & $27 / 0$ & Cold water (90 s) & $\begin{array}{l}\text { Contact } \\
\text { heat }(60 \mathrm{~s})\end{array}$ & No & [23] \\
\hline 5 & $23 \pm 5$ & $17 / 9$ & Cold water (60 s) & $\begin{array}{c}\text { Contact } \\
\text { heat }(30 \mathrm{~s})\end{array}$ & Yes & Unpublished \\
\hline 6 & $25 \pm 5$ & $9 / 19$ & Cold water (60 s) & $\begin{array}{c}\text { Contact } \\
\text { heat }(30 \mathrm{~s})\end{array}$ & No & Unpublished \\
\hline 7 & $25 \pm 3$ & $7 / 10$ & Cold water (60 s) & $\begin{array}{c}\text { Contact } \\
\text { heat }(30 \mathrm{~s})\end{array}$ & No & Unpublished \\
\hline
\end{tabular}

\subsection{Participants}

All studies were conducted in accordance with the Declaration of Helsinki and were approved by the ethics committee of the Ludwig-Maximilian University, Munich. Healthy 
participants were recruited by announcements on the university campus and gave written informed consent. Participants had to meet the following criteria (which apply to all our studies with healthy participants): (1) age $\geq 18$ years, (2) sufficient knowledge of German, (3) no severe internal, neurological or psychiatric conditions, (4) no history of chronic pain, (5) no alcohol, nicotine or drug abuse, (6) no regular medication (except hormonal contraception or thyroid hormones), (7) not pregnant or breastfeeding, (8) no acute pain and no use of pain medication within the previous $48 \mathrm{~h}$, (9) Beck's depression inventory score $<13$.

\subsection{Conditioned Pain Modulation}

The study data collected utilized three different CPM paradigms (combinations of test stimulus and conditioning stimulus).

The conditioning stimulus in all studies was immersion of the contralateral (in regard to the test stimulus) hand into a Styrofoam box filled with cold water for 60-120 s. Pain intensity ratings were collected on an 11-point numeric rating scale (NRS, $0=$ no pain, $10=$ most intense pain imaginable). According to the results of Granot [12], we aimed at a conditioning stimulus intensity that was clearly painful (usually $\geq 3$ on the NRS) but could be tolerated for the planned stimulus duration. To achieve this, in a pre-test, water temperature was individually adjusted starting at $10^{\circ} \mathrm{C}$.

Test stimuli were either painful heat ( $30 \mathrm{~s}$ or $60 \mathrm{~s}$ ) or electrical stimulation of the sural nerve.

Heat stimulation was applied via a thermode (Pathway system, Medoc, Israel) to the volar side of the forearm at a temperature individually tailored to evoke a pain intensity rating of approximately 6 on the NRS, resulting in temperatures of $46.3 \pm 1.2^{\circ} \mathrm{C}$, range: $43-49^{\circ} \mathrm{C}$. Heat pain intensity ratings were collected every $10 \mathrm{~s}$ for the stimulus duration. The heat stimulus was first presented in isolation (baseline) and then $30 \mathrm{~s}$ following the start of the conditioning stimulus. A $\geq 5$ min break was taken between baseline and conditioning measures and the thermode was shifted between measurements to avoid habituation.

Painful electrical stimulation of the sural nerve was performed as described previously [23,24]. Electrical stimuli were applied every $8-12 \mathrm{~s}$ for three consecutive $2 \mathrm{~min}$ blocks, each block containing 12 stimuli. Conditioning stimulus was present during the second block (120 s). Pain intensity rating of the test stimulus was collected at the end of each block as the average pain intensity of the last five stimuli.

CPM effect was calculated as the percentage difference between average test stimulus NRS rating at baseline (NRSts(baseline)) and during conditioning stimulation (NRSts(cond)), where a more negative result denotes a stronger CPM effect:

$$
\text { CPM effect }=\frac{\text { NRSts }(\text { cond })-\text { NRSts }(\text { baseline })}{\text { NRSts }(\text { baseline })}
$$

\subsection{Statistical Analysis}

All statistical analysis was performed in R [25]. $p<0.05$ (two-sided) was considered statistically significant.

Linear regression: linear regression analyses were performed using the $\operatorname{lm}()$ function of the stats package [25]. The linear regression models used for the cross-sectional population analysis of the relationship between CPM effect and age, sex, paradigm and conditioning stimulus pain or physical intensity were:

$$
\begin{gathered}
\text { CPM effect } \sim \mathrm{NRS}_{\text {cond }}+\text { age }+ \text { sex }+ \text { paradigm } \\
\text { CPM effect } \sim \text { temperature } \\
\text { cond }+ \text { age }+ \text { sex }+ \text { paradigm }
\end{gathered}
$$

$\mathrm{NRS}_{\text {cond }}$ describes the CS pain intensity on the NRS (0-10) immediately after the test

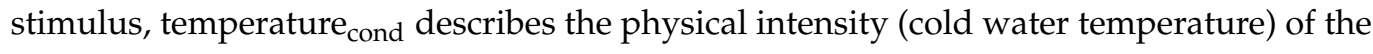


$\mathrm{CS}$ and paradigm describes the CPM paradigm as a factor. The three paradigms included were $60 \mathrm{~s}$ heat $/ 90 \mathrm{~s}$ cold (reference), $30 \mathrm{~s}$ heat $/ 60 \mathrm{~s}$ cold, and Electrical/120 s cold.

Mixed models: linear mixed model analysis was performed on the pooled repeated measures data using the lme4 [26] and car [27] packages in R. From the repeated measures population, we extracted those participants in which the CS pain intensity differed by at least 0.5 points on the NRS between measurements ( 85 participants, 184 observations) and those participants in which the CS physical intensity (temperature) differed by at least 0.5 ${ }^{\circ} \mathrm{C}$ between measurements (52 participants, 118 observations). Linear mixed models were constructed for the pain intensity rating variable, and the temperature variable subgroup using the lmer() function of lme4 [26]:

$$
\mathrm{CPM} \text { effect } \sim \mathrm{NRS}_{\text {cond }}+\text { age }+ \text { sex }+ \text { paradigm }+ \text { repeat }+(1 \text { I participant })
$$

$$
\text { CPM effect } \sim \text { temperature } \text { cond }+ \text { age }+ \text { sex }+ \text { paradigm }+ \text { repeat }+(1 \text { l participant })
$$

$\mathrm{CPM}$ effect, $\mathrm{NRS}_{\text {cond }}$ and temperature cond $_{\text {are }}$ as described above; paradigm describes the two CPM paradigms included in the dataset: $30 \mathrm{~s}$ heat $/ 60 \mathrm{~s}$ cold and Electrical/120s cold (reference). Repeat describes the measurement repeat (i.e., first, second, or third measurement for that participant). The random effect ( 1 | participant) allows for variable intercept for each participant. Significance of each fixed effect was tested using Wald's Chi-squared test implemented via the Anova() function of the car package [27].

Fixed vs. random effects variance contribution: in order to determine the interindividual variability of the CPM effect not explained by age and sex we calculated the percent variance in CPM effect explained as contributed by fixed and random effects. To do this we calculated the marginal and conditional coefficient of determination as described in Nakagawa et al. $[18,19]$ using the r.squared $G L M M()$ function of the MuMIn package [28] on the mixed models described above. Marginal $\mathrm{R}^{2}$ describes the variance explained by all fixed effects and conditional $R^{2}$ describes the variance explained by both fixed and random effects combined. Variance explained by inter-individual variability was calculated by subtracting the marginal from the conditional $\mathrm{R}^{2}$.

Variance contribution of the fixed effects: in order to determine the relative contribution of each fixed effect factor to the variance in the CPM effect, the calc.relimp () function of the relaimpo package was used [29]. We utilized the "lmg" option of $\mathrm{R}^{2}$ variance decomposition, which averages the $\mathrm{R}^{2}$ contribution of each factor over all orderings as described by Lindeman, Merenda and Gold [21] and Chevan and Sutherland [20].

As calc.relimp () cannot handle mixed model input, we constructed the following linear models using the $\operatorname{lm}($ ) function to reflect the fixed effects of the mixed model 3 and 4 and used them as input to the calc.relimp () function:

$$
\begin{gathered}
\text { CPM effect } \sim \mathrm{NRS}_{\text {cond }}+\text { age }+ \text { sex }+ \text { paradigm }+ \text { repeat } \\
\text { CPM effect } \sim \text { temperature } \text { cond }_{\text {cog }}+\text { agex }+ \text { paradigm }+ \text { repeat }
\end{gathered}
$$

\section{Results}

\subsection{Cross-Sectional Analysis}

The mean age of the cross-sectional sample was $29 \pm 11$ ( $n=171,64$ women). The average CPM effect was significant $(p<0.001)$ and amounted to $-16.9 \pm 23.9 \%$. The average pain rating of the conditioning stimulus was $4.5 \pm 1.8$ (range: 1.0-9.0) on the NRS and the average temperature of the conditioning stimulus was $7.9 \pm 3.4^{\circ} \mathrm{C}$ (range: 0.1-16.2).

Multiple linear regression was calculated to predict CPM effect from participant age, sex, CPM protocol and either CS pain or physical intensity (models 1 and 2, Table 2). There was no significant relation of the CPM effect with CS pain intensity ( $\mathrm{NRS}_{\text {cond }}$ ) or CS physical intensity (temperature cond $_{\text {) }}$ (Figure 1). There also was no significant relation of age, sex or CPM paradigm with the CPM effect. Proportions of variance in CPM effect explained by all predictors together were low (1.1\% for model 1 and $1.9 \%$ for model 2$)$. Results for the individual predictors are given in Table 2. 
Table 2. Results of linear regression analysis of the cross-sectional data $(n=171)$. See Methods for construction of models 1 and $3 . \mathrm{NRS}_{\text {cond' }}$ CS pain intensity rating on the NRS [0-10]. Temperature cond $_{\text {, }}$ temperature of the CS (cold water bath). Fixed effects of sex and paradigm were compared to a reference (male and $30 \mathrm{~s}$ heat $60 \mathrm{~s}$ cold, respectively).

\begin{tabular}{|c|c|c|c|c|c|}
\hline Model & Predictor & Estimate & Std. Error & $p$-Value & Multiple $\mathbf{R}^{2}$ \\
\hline \multirow{6}{*}{ Model 1} & Intercept & -17.385 & 9.933 & 0.082 & \multirow{6}{*}{0.0109} \\
\hline & $\mathrm{NRS}_{\text {cond }}$ & -0.916 & 1.101 & 0.407 & \\
\hline & Age & -0.053 & 0.288 & 0.854 & \\
\hline & Sex & 4.181 & 4.080 & 0.307 & \\
\hline & $30 \mathrm{~s}$ heat $/ 60 \mathrm{~s}$ cold & 3.643 & 8.853 & 0.681 & \\
\hline & Electrical/120 s cold & -1.190 & 4.988 & 0.812 & \\
\hline \multirow{6}{*}{ Model 2} & Intercept & -27.890 & 10.237 & 0.007 & \multirow{6}{*}{0.0194} \\
\hline & Temperature $_{\text {cond }}$ & 0.866 & 0.594 & 0.147 & \\
\hline & Age & -0.102 & 0.288 & 0.723 & \\
\hline & Sex & 4.818 & 4.096 & 0.242 & \\
\hline & Heat $30 \mathrm{~s} /$ Cold $60 \mathrm{~s}$ & 1.825 & 8.715 & 0.834 & \\
\hline & Electrical/120s cold & 0.470 & 5.141 & 0.927 & \\
\hline
\end{tabular}
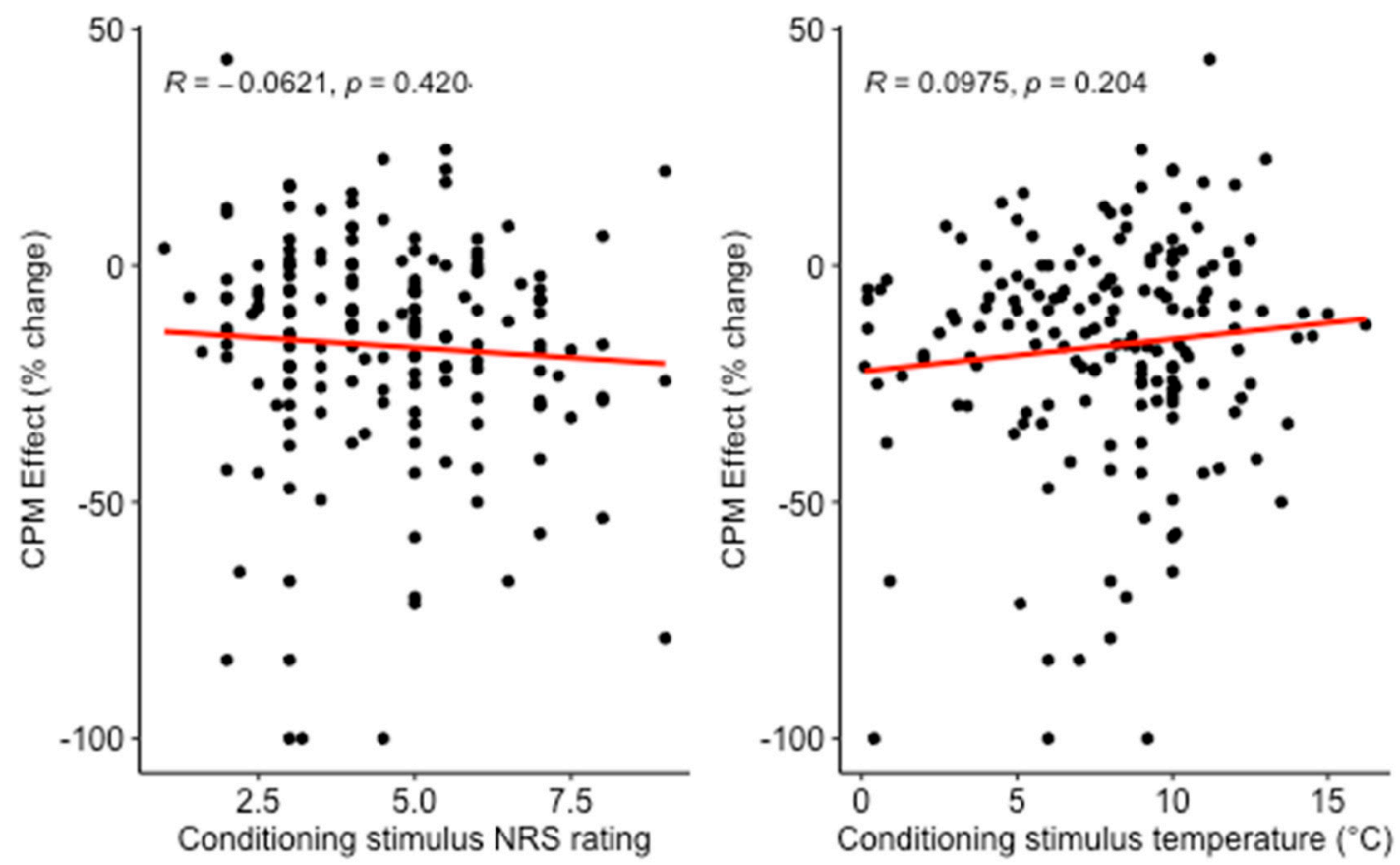

Figure 1. Relation between CPM effect and conditioning stimulus pain intensity or conditioning stimulus temperature in the cross-sectional analysis. Conditioning stimulus was hand immersion in cold water. More negative CPM effect designates a larger reduction of test pain rating by the conditioning stimulus.

\subsection{Repeated Measures Analysis of Linear Mixed Models}

The average CPM effect in the repeated measures sample (Model 3: 184 observations, Model 4: 118 observations) was significant (both $p<0.001$ ) and amounted to $-17.6 \pm 24.6 \%$ and $-16.9 \pm 21.2 \%$, respectively. Mean difference in NRS rating of CS between observations in Model 3 was $1.9 \pm 1.2$. Mean difference in CS temperature between observations in Model 4 was $4.3 \pm 2.6^{\circ} \mathrm{C}$.

Linear mixed models were constructed in order to analyze the contributions of the different fixed effects to the CPM effect (Table 3). A larger (i.e., more negative) CPM effect was significantly related to a lower CS temperature $(p=0.001,-1.5 \%$ change in CPM effect per ${ }^{\circ} \mathrm{C}$ temperature decrease) in Model 4. 
The remaining relations were all non-significant (Table 3): in Model 3, CPM effect increased (i.e., was more negative) by $-1.5 \%$ per NRS point with increasing CS pain intensity $(p=0.159)$. CPM effect decreased non significantly by $0.26 \%$ per year of age in both models. CPM effect in women was 2.5\% lower in Model 3, and 1.9\% larger in Model 4 compared to men (both n.s.). The $30 \mathrm{~s}$ heat $/ 60 \mathrm{~s}$ cold protocol produced a 7.8\% (Model 3) and $8.5 \%$ (Model 4) larger CPM effect than the electrical/120 s cold protocol (both n.s.).

Table 3. Relation between CPM effect and CS pain intensity or physical intensity (temperature) in the repeated measures analysis. Linear mixed effect analysis of Model 3 ( $n=85$ participants, 184 observations) and Model 4 ( $\mathrm{n}=52$ participants, 118 observations), see Methods for model specification. $p$-values were obtained by Wald's Chi-Square test on the fitted mixed models. NRScond, CS pain intensity rating on the NRS [0-10]. Temperaturecond, temperature of the CS (cold water bath). Paradigm, $30 \mathrm{~s}$ heat/60 s cold as opposed to Electrical/cold120s (reference). Repeat refers to the measurement repeat and is used to control for order of measurement. Significant effects are marked in bold.

\begin{tabular}{|c|c|c|c|c|c|}
\hline Model & Predictor & Estimate & $\begin{array}{l}\text { Std. } \\
\text { Error }\end{array}$ & $p$-Value & $\begin{array}{l}\text { REML Criterium at } \\
\text { Convergence }\end{array}$ \\
\hline \multirow{6}{*}{ Model 3} & Intercept & -11.685 & 13.461 & - & \multirow{6}{*}{1649.6} \\
\hline & $\mathrm{NRS}_{\text {cond }}$ & -1.485 & 1.056 & 0.159 & \\
\hline & Age & 0.257 & 0.388 & 0.506 & \\
\hline & Sex & 2.452 & 4.402 & 0.578 & \\
\hline & Paradigm & -7.815 & 4.808 & 0.104 & \\
\hline & Repeat & -2.617 & 2.637 & 0.321 & \\
\hline \multirow{6}{*}{ Model 4} & Intercept & -21.712 & 13.935 & - & \multirow{6}{*}{1015.8} \\
\hline & Temperature $_{\text {cond }}$ & 1.532 & 0.482 & 0.002 & \\
\hline & Age & 0.258 & 0.428 & 0.546 & \\
\hline & Sex & -1.860 & 4.831 & 0.700 & \\
\hline & Paradigm & -8.468 & 4.910 & 0.085 & \\
\hline & Repeat & -3.073 & 2.343 & 0.190 & \\
\hline
\end{tabular}

\subsection{Repeated Measures Analysis: Decomposition of Explained Variance}

To determine the relative variance explained by fixed effects vs. inter-individual differences we decomposed the total $\mathrm{R}^{2}$ into conditional (fixed effects) and marginal (fixed effects + inter-individual factors) $\mathrm{R}^{2}$. Conditional $\mathrm{R}^{2}$ and marginal $\mathrm{R}^{2}$ were $3.4 \%$ and $27.4 \%$ in Model 3, and $11.5 \%$ and $45.8 \%$ in Model 4 , respectively. Therefore, in Model 3, all fixed effects together explained $3.4 \%$ of the variance in the CPM effect, while the remaining inter-individual differences explained 24.0\%. In Model 4, all fixed effects together explained $11.5 \%$ of the variance in the $\mathrm{CPM}$ effect and remaining inter-individual differences explained $34.3 \%$.

Finally, in order to further decompose the variance explained by the different fixed effects, we constructed linear models including only the fixed effects (Models 5 and 6, Table 4). CS pain intensity explained $0.7 \%$ of the variance (model 5) while CS physical intensity (cold water temperature) explained 4.7\% (Model 6). The type of CPM paradigm used explained $1.5 \%$ and $3.0 \%$ of the variance in Model 5 and 6, respectively. Age, sex and measurement repeat made only small contributions to the explained variance ( $<1 \%$ each). Variance breakdown of the significant model (Model 4) and the relative variances of its fixed effect are seen in Figure 2. It is important to note that the fixed effects' variance in Models 5 and 6 does not sum to the variance explained by fixed effects in Models 3 and 4, respectively. This is due to the fact that the models used differ, resulting in slightly different fits. Additionally the statistical methods used for variance decomposition in the two types of analysis are different, which will further lead to discrepancies. 
Table 4. Variance in the CPM effect explained by fixed effects, including CS pain and physical intensity in the repeated measures analysis. Model 5: $\mathrm{n}=85$ participants, 184 observations; model 6: $\mathrm{n}=52$ participants, 118 observations, see Methods for model specifications. $\mathrm{R}^{2}$ indicates the total variance explained by each individual predictor as calculated by the calc.relimp () (relaimpo package) in R. NRScond, pain intensity rating of conditioning stimulus on the NRS [0-10]. Temperaturecond, temperature of the conditioning stimulus (cold water bath). Paradigm, $30 \mathrm{~s}$ heat $/ 60 \mathrm{~s}$ cold as opposed to Electrical/120 s cold (reference). Repeat refers to the measurement repeat and is used to control for order of measurement.

\begin{tabular}{cccc}
\hline & Model 5 & \multicolumn{2}{c}{ Model 6 } \\
\hline Predictor & $\mathrm{R}^{2}$ & Predictor & $\mathrm{R}^{2}$ \\
NRS $_{\text {cond }}$ & 0.00681 & Temperature & 0.04650 \\
Age & 0.00438 & Age & 0.00886 \\
Sex & 0.00559 & Sex & 0.00091 \\
Paradigm & 0.01491 & Paradigm & 0.03010 \\
Repeat & 0.00325 & Repeat & 0.00871 \\
\hline
\end{tabular}

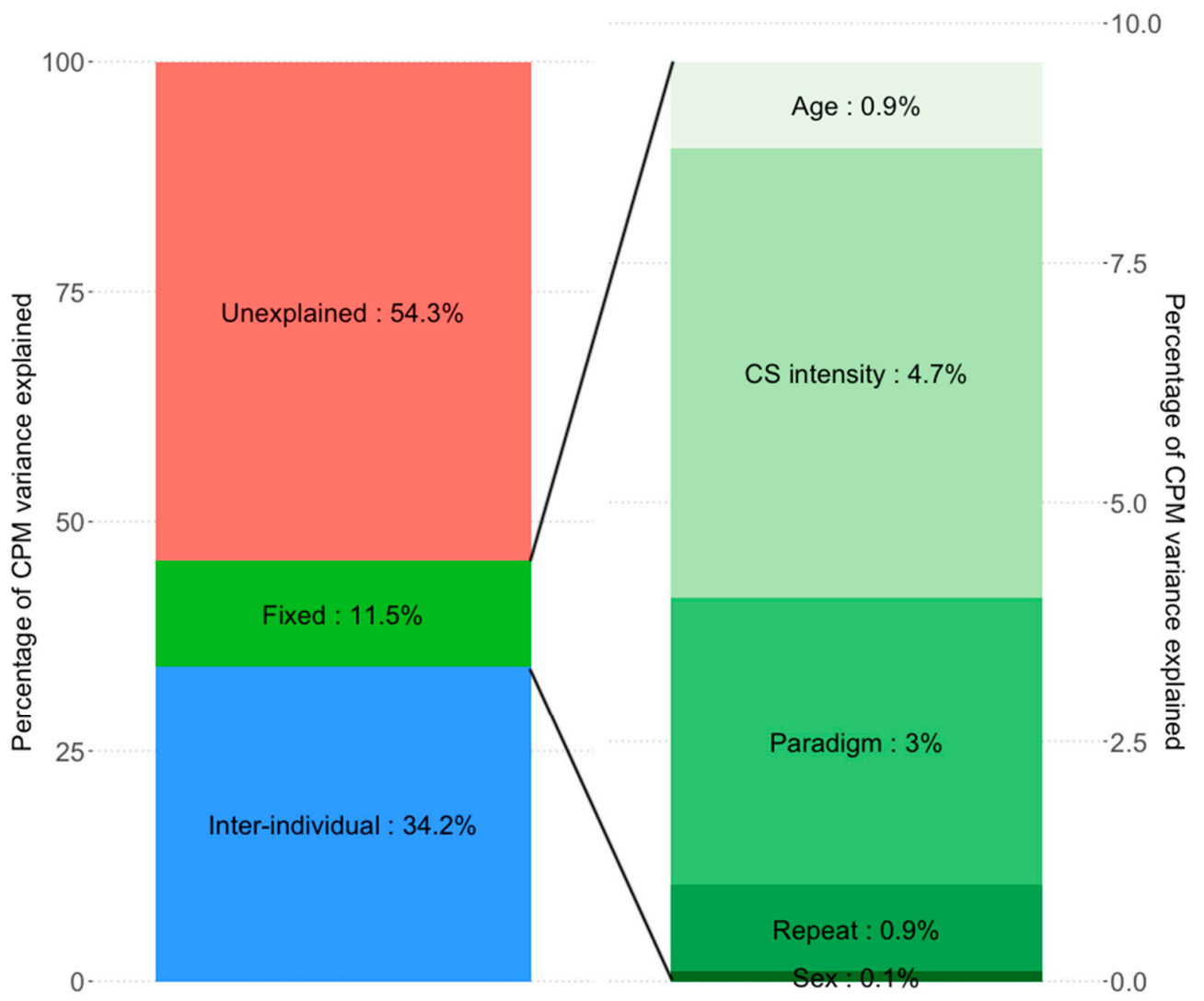

Figure 2. Proportions of variance in CPM explained by the CS physical intensity models (models 4 and 6). Inter-individual differences explain substantially more CPM variance than the fixed effects of age, sex, CS intensity or the nuisance regressors CPM paradigm or measurement repeat (34.2\% vs. $11.5 \%$, respectively). Of the fixed effects, CS intensity explains the most variance $(4.65 \%)$, followed by CPM paradigm (3.01\%). Age $(0.89 \%)$, Sex $(0.09 \%)$, and measurement repeat $(0.87 \%)$ explain negligible amounts. Due to different model types (model 4: linear mixed effects model; Model 6: multiple linear regression model) and different statistical methods needed to estimate partial variance explained, the sum of fixed effects variance explained in Model 6 does not equal exactly the estimated variance of combined fixed effects in Model 4. 


\section{Discussion}

Main results of the present study were:

(i) In a large cross-sectional analysis, neither CS physical intensity nor CS pain intensity predicted the CPM effect. In contrast, in a repeated measures analysis, CS physical intensity, but not CS pain intensity predicted the CPM effect.

(ii) Inter-individual differences explained a large proportion of $\mathrm{CPM}$ variance $(24.0 \%$ to $34.2 \%$ ) while all fixed effects together (CS pain or physical intensity, age, sex, CPM paradigm, measurement repeat) predicted only $3.4 \%$ to $11.5 \%$ of CPM variance.

\subsection{Conditioning Stimulus Physical Intensity and Pain Intensity}

Previous results on the dependence of CPM magnitude on CS intensity are inconsistent, ranging from no effect [11-13] to a significantly increased CPM effect with higher CS intensity [14-16]. Our study confirms and extends previous cross-sectional results [12] showing no significant relation between CPM magnitude and either CS physical intensity or CS pain rating in a large cross-sectional sample $(n=171)$. In contrast, the repeated measures analysis revealed a significant relation between CPM magnitude and CS physical intensity, while the relation with CS pain intensity remained non-significant. This raises two interesting points.

First, as CPM is a psychophysical measure, one could assume that the subjective pain experience would have a larger influence on the CPM effect than the CS physical intensity. Indeed, some studies have shown a relation between CS pain intensity and CPM magnitude $[15,16]$. In addition, placebo-induced reduction of perceived CS pain was related to a reduced CPM effect [30] and CS-induced supraspinal activation correlated with CPM magnitude [31]. On the other hand, some processes underlying CPM seem to be independent of the subjective pain experience [32]. In spite of the above cited supraspinal influences, a spino-bulbo-spinal pathway is thought to be the main circuitry responsible for CPM [33,34]. This may be one possible explanation for CS physical intensity being a larger determinant than CS pain intensity. Consistently, some previous studies have shown a relation between CS physical intensity and CPM effect $[14,35]$. However, since CS physical and pain intensity are highly correlated, only studies that investigate both parameters over a range of different values will be able to show which correlation is larger. The present study conducted such a direct comparison and found a preferential relation with CS physical intensity. Notably, it may be both a strength and a limitation of the present study that variability in CS physical and/or pain intensity was mostly random and not due to a dedicated study design. This point would clearly merit further investigation, systematically and independently varying both CS physical and pain intensity, ideally over more than two to three observations per subject.

Second, the significant relation between CPM magnitude and CS physical intensity was detected in the repeated measures but not in the cross-sectional analysis. This suggests that within a given subject, there is a dependence of CPM magnitude on CS physical intensity, which however is small compared to inter-individual variability in the CPM effect. Analysis of explained variance indeed showed that the variance due to interindividual differences is much larger than the variance explained by CS physical intensity (see below).

\subsection{Age, Sex, Measurement Eepeat and CPM Paradigm}

While some previous findings have suggests less efficient CPM with increasing age $[7,8,36]$, we did not find such a relationship. This may be due to the limited age range present in our dataset, as most of our participants were young. We also found no difference in CPM effect between men and women, which is consistent with some previous findings [37,38], but larger CPM effects in men compared to women have also been reported [6,39]. We found no significant effect of the CPM paradigm used, suggesting no inherent difference between paradigms. However, we did not aim to investigate the effect of paradigm and included it as a regressor solely to control for any potential 
paradigm-related differences. Our data stemmed from only three different paradigms, and all used cold pain as CS. Measurement repeat was also included for control, not revealing any significant effects.

\subsection{Variance Explained by Inter-Individual Differences vs. Fixed Variables}

To our knowledge, our study is the first to estimate the relative importance of intraindividual differences (other than age or sex) vs. fixed variables for the CPM effect. By including only participant as random effect, we could estimate only the variance attributed to each individual participant, i.e., the inter-individual contribution to CPM variance. It resulted that the variance explained by inter-individual differences was large ( $24.0 \%$ to $34.2 \%$ ) compared to that of all fixed effects combined (3.4\% to $11.5 \%)$. Among the fixed effects, while the contribution of CS pain intensity, age, sex and measurement repeat were all $<1 \%$, only CS physical intensity $(4.7 \%)$ and CPM paradigm $(1.5 \%$ to $3.0 \%)$ made a somewhat larger contribution. This again raises two important points for discussion.

First, the large effect of inter-individual differences provides an important basis for recent attempts to use inter-individual differences as a predictor for acute or chronic pain states [3,40-42]. In comparison, methodological effects such as CPM paradigm and CS stimulus intensity explain much less variance. Even the contribution of commonly included factors age and sex seems to be small in comparison, with the caveat that our data were not optimally suited to detect age effects.

Second, as suggested above, the large inter-individual differences may be the reason why the relation between CS intensity and CPM effect might be difficult to detect in crosssectional studies. Such inter-individual differences could be due to genetic, epigenetic, developmental and/or behavioral differences. Indeed, Lindstedt et al. [43] showed that genetic variation in a serotonin transporter gene is related to CPM magnitude. Cardiovascular reactivity to pain also seems to be related to CPM magnitude [44]. Psychological traits such as anxiety, depression, or catastrophizing might also contribute to inter-individual differences. Although a meta-analysis by Nahman-Averbuch et al. [9] found no link between psychological traits and overall CPM effect in healthy subjects, they did show a modality-specific relation with psychological scores of depression, anxiety and catastrophizing. Acute changes in catastrophizing and mood have been shown to influence endogenous pain inhibition $[45,46]$. Moreover, the role of psychological factors might be more important in clinical populations who tend to have more pronounced psychological traits. These considerations give ample room for further studies to dissect the nature of individual differences in CPM magnitude., e.g., twins studies for the role of genetic differences, and studies looking at inter-individual differences in clinical populations using a similar methodology, including psychological factors, while accounting for inter-individual differences as random factors to discern how much variance these traits account for.

Nonetheless, standardized methods are clearly desirable, and controlling for interindividual differences in repeated measures designs may allow researchers to detect other, smaller contributing factors that would otherwise go undetected.

\subsection{Unexplained Variance}

In the present analysis, with $3.4 \%$ to $11.5 \%$ accounted for by fixed effects and $24.0 \%$ to $34.3 \%$ by inter-individual variability; this leaves $54.2 \%$ to $72.6 \%$ of the variance in CPM magnitude unexplained. Multiple factors may contribute to this. Test-retest reliability of CPM yields intraclass correlation coefficients between 0.21 and $0.82[14,47,48]$ (reviewed in [49]), showing that even under constant experimental conditions, there is still a significant amount of variability between measurements. This variability may be explained in part by measurement error, which is expected when dealing with subjective pain reports. In addition, there might also be something such as the "daily form" of the subject., e.g., transient psychological states such as acute anxiety or catastrophizing might influence CPM magnitude, especially in clinical populations. Additionally, tiredness, physical activity, menstrual phase, distraction, and previous experiences and expectations might 
influence the CPM effect differently between sessions. Indeed, it has been shown that factors such as distraction, catastrophizing and even voluntary mental strategies can acutely change the activity of endogenous pain inhibitory systems [24,45,50]. In addition, experimental conditions not considered in the present study, such as time of the day, gender and personality of the experimenter, or use of pain ratings compared to pain thresholds for the test stimulus might be factors that remain to be investigated.

\subsection{Strengths and Limitations}

The major strengths of our study are (1) the inclusion of a relatively large sample that allowed the comparison of cross-sectional and repeated measures effects for both CS physical and pain intensity, and (2) the use of new techniques for variance decomposition that allowed the estimation of the variance contribution of inter-individual differences and fixed effects within the same model. There are also some major limitations. First, our analysis did not include a comprehensive sample of different CPM paradigms. Therefore, it remains to be confirmed if these results translate to other CPM paradigms, especially those which assess pain thresholds instead of pain ratings for the test stimulus. Second, in our repeated measures analysis the majority of our participants only had two repeats. Repeating our analysis using multiple CS intensities and pain levels would potentially lead to more robust results. Third, our repeat analysis did not include a broad age-range, possibly precluding detection of an age effect. Lastly, our data is derived exclusively from healthy participants. It remains to be determined if our findings can be applied to clinical populations, such as chronic pain patients or patients undergoing painful medical procedures.

\section{Conclusions}

The present data emphasize the role of inter-individual differences in CPM magnitude, providing a basis for investigating these differences in clinical populations and using CPM as a predictive tool to individualize medicine by giving insight into the individuals' endogenous pain modulation system. They also show that in comparison, CS intensity makes a minor contribution to CPM magnitude. In repeated measures designs, to further reduce methodological effects on CPM measurement, keeping CS physical intensity constant seems to be more important than CS pain intensity.

Author Contributions: Conceptualization, P.G., A.I., K.W., R.R.; methodology, P.G. and R.R.; formal analysis, P.G.; data curation, P.G., A.I., K.W.; writing-original draft preparation, P.G. and R.R.; writing-review and editing, P.G., A.I., K.W., R.R.; visualization, P.G.; supervision, R.R.; project administration, R.R. All authors have read and agreed to the published version of the manuscript.

Funding: This work was funded by the German Research Association (DFG) via the "RTG2175: Perception in context and its Neural Basis" and the Graduate School of Systemic Neurosciences.

Institutional Review Board Statement: All studies involved were conducted according to the guidelines of the Declaration of Helsinki, and approved by the local Ethics Committees at the University Hospital, Ludwig-Maximilian University Munich, Munich, Germany.

Informed Consent Statement: Informed consent was obtained from all subjects involved in the study.

Data Availability Statement: Data is available upon reasonable request from the corresponding author.

Acknowledgments: The authors would like to thank Laura Alt, Regina Stacheneder and Stefanie Krafft. for their contribution to data acquisition, Virginia Flanagin. for discussion on data analysis and all volunteers for their participation in the various studies.

Conflicts of Interest: The authors declare no conflict of interest. 


\section{References}

1. Yarnitsky, D. Conditioned pain modulation (the diffuse noxious inhibitory control-like effect): Its relevance for acute and chronic pain states. Curr. Opin. Anaesthesiol. 2010, 23, 611-615. [CrossRef] [PubMed]

2. Lewis, G.N.; Rice, D.A.; McNair, P.J. Conditioned pain modulation in populations with chronic pain: A systematic review and meta-analysis. J. Pain 2012, 13, 936-944. [CrossRef] [PubMed]

3. Nir, R.-R.; Yarnitsky, D. Conditioned pain modulation. Curr. Opin. Support. Palliat. Care 2015, 9, 131-137. [CrossRef] [PubMed]

4. Yarnitsky, D.; Crispel, Y.; Eisenberg, E.; Granovsky, Y.; Ben-Nun, A.; Sprecher, E.; Best, L.-A.; Granot, M. Prediction of chronic post-operative pain: Pre-operative DNIC testing identifies patients at risk. Pain 2008, 138, 22-28. [CrossRef]

5. Staud, R.; Robinson, M.E.; Vierck, C.J.; Price, D.D. Diffuse noxious inhibitory controls (DNIC) attenuate temporal summation of second pain in normal males but not in normal females or fibromyalgia patients. Pain 2003, 101, 167-174. [CrossRef]

6. Arendt-Nielsen, L.; Sluka, K.A.; Nie, H.L. Experimental muscle pain impairs descending inhibition. Pain 2008, 140 , 465-471. [CrossRef]

7. Riley, J.L.; King, C.D.; Wong, F.; Fillingim, R.B.; Mauderli, A.P. Lack of endogenous modulation and reduced decay of prolonged heat pain in older adults. Pain 2010, 150, 153-160. [CrossRef]

8. Larivière, M.; Goffaux, P.; Marchand, S.; Julien, N. Changes in pain perception and descending inhibitory controls start at middle age in healthy adults. Clin. J. Pain 2007, 23, 506-510. [CrossRef]

9. Nahman-Averbuch, H.; Nir, R.R.; Sprecher, E.; Yarnitsky, D. Psychological factors and conditioned pain modulation: A metaanalysis. Clin. J. Pain 2016, 32, 541-554. [CrossRef]

10. Pud, D.; Granovsky, Y.; Yarnitsky, D. The methodology of experimentally induced diffuse noxious inhibitory control (DNIC)-like effect in humans. Pain 2009, 144, 16-19. [CrossRef]

11. Nir, R.R.; Granovsky, Y.; Yarnitsky, D.; Sprecher, E.; Granot, M. A psychophysical study of endogenous analgesia: The role of the conditioning pain in the induction and magnitude of conditioned pain modulation. Eur. J. Pain 2011, 15, 491-497.

12. Granot, M.; Weissman-Fogel, I.; Crispel, Y.; Pud, D.; Granovsky, Y.; Sprecher, E.; Yarnitsky, D. Determinants of endogenous analgesia magnitude in a diffuse noxious inhibitory control (DNIC) paradigm: Do conditioning stimulus painfulness, gender and personality variables matter? Pain 2008, 136, 142-149. [CrossRef] [PubMed]

13. Nahman-Averbuch, H.; Yarnitsky, D.; Granovsky, Y.; Gerber, E.; Dagul, P.; Granot, M. The role of stimulation parameters on the conditioned pain modulation response. Scand. J. Pain 2013, 4, 10-14. [CrossRef] [PubMed]

14. Graven-Nielsen, T.; Izumi, M.; Petersen, K.K.; Arendt-Nielsen, L. User-independent assessment of conditioning pain modulation by cuff pressure algometry. Eur. J. Pain 2017, 21, 552-561. [CrossRef] [PubMed]

15. Oono, Y.; Wang, K.; Svensson, P.; Arendt-Nielsen, L. Conditioned pain modulation evoked by different intensities of mechanical stimuli applied to the craniofacial region in healthy men and women. J. Orofac. Pain 2011, 25, 364-375.

16. Smith, A.; Pedler, A. Conditioned pain modulation is affected by occlusion cuff conditioning stimulus intensity, but not duration Eur. J. Pain 2018, 22, 94-102. [CrossRef]

17. Willer, J.C.; Roby, A.; le Bars, D. Psychophysical and electrophysiological approaches to the pain-relieving effects of heterotopic nociceptive stimuli. Brain 1984, 107, 1095-1112. [CrossRef]

18. Nakagawa, S.; Schielzeth, H. A general and simple method for obtaining R2 from generalized linear mixed-effects models. Methods Ecol. Evol. 2013, 4, 133-142. [CrossRef]

19. Nakagawa, S.; Johnson, P.C.D.; Schielzeth, H. The coefficient of determination R2 and intra-class correlation coefficient from generalized linear mixed-effects models revisited and expanded. J. R. Soc. Interface 2017, 14, 20170213. [CrossRef]

20. Chevan, A.; Sutherland, M. Hierarchical partitioning. Am. Stat. 1991, 45, 90-96.

21. Lindeman, R.H.; Merenda, P.F.; Gold, R.Z. Introduction to Bivariate and Multivaraite Analysis; Foresman and Company: Glenview, IL, USA, 1980.

22. Alt, L.K.; Wach, K.; Liebler, E.J.; Straube, A.; Ruscheweyh, R. A randomized sham-controlled cross-over study on the short-term effect of non-invasive cervical vagus nerve stimulation on spinal and supraspinal nociception in healthy subjects. Headache 2020, 60, 1616-1631. [CrossRef]

23. Krafft, S.; Göhmann, H.D.; Sommer, J.; Straube, A.; Ruscheweyh, R. Learned control over spinal nociception in patients with chronic back pain. Eur. J. Pain 2017, 21, 1538-1549. [CrossRef] [PubMed]

24. Ruscheweyh, R.; Kreusch, A.; Albers, C.; Sommer, J.; Marziniak, M. The effect of distraction strategies on pain perception and the nociceptive flexor reflex (RIII reflex). Pain 2011, 152, 2662-2671. [CrossRef] [PubMed]

25. R Core Team R: A Language and Environment for Statistical Computing; R Foundation for Statistical Computing: Vienna, Austria, 2020.

26. Bates, D.; Maechler, M.; Bolker, B.; Walker, S. Fitting Linear Mixed-Effects Models Using lme4. J. Stat. Softw. 2015, 67, 1-48. [CrossRef]

27. Fox, J.; Weisberg, S. An \{R\} Companion to Applied Regression, 3rd ed.; Sage: Thousand Oaks, CA, USA, 2019.

28. Bartón, K. MuMIn: Multi-Model Inference. 2020. Available online: https:/ / cran.r-project.org/ package=MuMIn (accessed on 6 September 2021).

29. Grömping, U. Relative importance for linear regression in R: The package relaimpo. J. Stat. Softw. 2006, 17, 1-27. [CrossRef]

30. Nir, R.R.; Yarnitsky, D.; Honigman, L.; Granot, M. Cognitive manipulation targeted at decreasing the conditioning pain perception reduces the efficacy of conditioned pain modulation. Pain 2012, 153, 170-176. [CrossRef] [PubMed] 
31. Sprenger, C.; Bingel, U.; Büchel, C. Treating pain with pain: Supraspinal mechanisms of endogenous analgesia elicited by heterotopic noxious conditioning stimulation. Pain 2011, 152, 428-439. [CrossRef]

32. Lautenbacher, S.; Roscher, S.; Strian, F. Inhibitory effects do not depend on the subjective experience of pain during heterotopic noxious conditioning stimulation (HNCS): A contribution to the psychophysics of pain inhibition. Eur. J. Pain 2002, 6, 365-374. [CrossRef]

33. le Bars, D.; Dickenson, A.H.; Besson, J.M. Diffuse noxious inhibitory controls (DNIC). I. Effects on dorsal horn convergent neurones in the rat. Pain 1979, 6, 283-304. [CrossRef]

34. Youssef, A.M.; Macefield, V.G.; Henderson, L.A. Pain inhibits pain; human brainstem mechanisms. Neuroimage 2016, 124, 54-62. [CrossRef]

35. Tousignant-Laflamme, Y.; Pagé, S.; Goffaux, P.; Marchand, S. An experimental model to measure excitatory and inhibitory pain mechanisms in humans. Brain Res. 2008, 1230, 73-79. [CrossRef]

36. Edwards, R.R.; Ness, T.J.; Weigent, D.A.; Fillingim, R.B. Individual differences in diffuse noxious inhibitory controls (DNIC): Association with clinical variables. Pain 2003, 106, 427-437. [CrossRef]

37. Rosén, A.; Feldreich, A.; Dabirian, N.; Ernberg, M. Effect of heterotopic noxious conditioning stimulation on electrical and pressure pain thresholds in two different anatomical regions. Acta Odontol. Scand. 2008, 66, 181-188. [CrossRef] [PubMed]

38. Treister, R.; Eisenberg, E.; Gershon, E.; Haddad, M.; Pud, D. Factors affecting-And relationships between-Different modes of endogenous pain modulation in healthy volunteers. Eur. J. Pain 2010, 14, 608-614. [CrossRef]

39. Ge, H.Y.; Madeleine, P.; Arendt-Nielsen, L. Sex differences in temporal characteristics of descending inhibitory control: An evaluation using repeated bilateral experimental induction of muscle pain. Pain 2004, 110, 72-78. [CrossRef] [PubMed]

40. Gruener, H.; Zeilig, G.; Gaidukov, E.; Rachamim-Katz, O.; Ringler, E.; Blumen, N.; Engel-Haber, E.; Defrin, R. Biomarkers for predicting central neuropathic pain occurrence and severity after spinal cord injury: Results of a long-term longitudinal study. Pain 2020, 161, 545-556. [CrossRef]

41. Müller, M.; Bütikofer, L.; Andersen, O.K.; Heini, P.; Arendt-Nielsen, L.; Jüni, P.; Curatolo, M. Cold pain hypersensitivity predicts trajectories of pain and disability after low back surgery: A prospective cohort study. Pain 2021, 162, 184-194. [CrossRef] [PubMed]

42. Yarnitsky, D.; Granot, M.; Granovsky, Y. Pain modulation profile and pain therapy: Between pro- and antinociception. Pain 2014, 155, 663-665. [CrossRef]

43. Lindstedt, F.; Berrebi, J.; Greayer, E.; Lonsdorf, T.B.; Schalling, M.; Ingvar, M.; Kosek, E. Conditioned pain modulation is associated with common Polymorphisms in the serotonin transporter gene. PLoS ONE 2011, 6, e18252. [CrossRef]

44. Chalaye, P.; Devoize, L.; Lafrenaye, S.; Dallel, R.; Marchand, S. Cardiovascular influences on conditioned pain modulation. Pain 2013, 154, 1377-1382. [CrossRef]

45. Ruscheweyh, R.; Albers, C.; Kreusch, A.; Sommer, J.; Marziniak, M. The effect of catastrophizing self-statements on pain perception and the nociceptive flexor reflex (RIII reflex). Clin. J. Pain 2013, 29, 725-732. [CrossRef]

46. Ruscheweyh, R.; Weinges, F.; Schiffer, M.; Bäumler, M.; Feller, M.; Krafft, S.; Straube, A.; Sommer, J.; Marziniak, M. Control over spinal nociception as quantified by the nociceptive flexor reflex (RIII reflex) can be achieved under feedback of the RIII reflex. Eur. J. Pain 2015, 19, 480-489. [CrossRef] [PubMed]

47. Granovsky, Y.; Miller-Barmak, A.; Goldstein, O.; Sprecher, E.; Yarnitsky, D. CPM test-retest reliability: "Standard" vs "single test-stimulus" protocols. Pain Med. 2016, 17, 521-529. [CrossRef] [PubMed]

48. Jurth, C.; Rehberg, B.; von Dincklage, F. Reliability of subjective pain ratings and nociceptive flexion reflex responses as measures of conditioned pain modulation. Pain Res. Manag. 2014, 19, 93-96. [CrossRef]

49. Kennedy, D.L.; Kemp, H.I.; Ridout, D.; Yarnitsky, D.; Rice, A.S.C. Reliability of conditioned pain modulation: A systematic review. Pain 2016, 157, 2410-2419. [CrossRef] [PubMed]

50. Rhudy, J.L.; Williams, A.E.; McCabe, K.M.; Nguyên, M.A.T.V.; Rambo, P. Affective modulation of nociception at spinal and supraspinal levels. Psychophysiology 2005, 42, 579-587. [CrossRef] [PubMed] 